\title{
In Vivo Spinal Distribution of Cy5.5 Fluorescent Dye after Injection via the Lateral Ventricle and Cisterna Magna in Rat Model
}

\author{
Kee-Hang Lee, Ph.D., ${ }^{1-3, *}$ Hyun Nam, Ph.D., ${ }^{2-4, *}$ Jeong-Seob Won, B.S., ${ }^{1-3}$ Ji-Yoon Hwang, B.S., ${ }^{2-4}$ Hye Won Jang, M.D., Ph.D., ${ }^{5}$ \\ Sun-Ho Lee, M.D., Ph.D., ${ }^{1,3,4}$ Kyeung Min Joo, M.D., Ph.D. ${ }^{1-3,6}$ \\ Department of Health Sciences and Technology, SAIHST, Sungkyunkwan University, Seoul, Korea \\ Single Cell Network Research Center, ${ }^{2}$ Sungkyunkwan University School of Medicine, Suwon, Korea \\ Stem Cell and Regenerative Medicine Center, ${ }^{3}$ Research Institute for Future Medicine, Samsung Medical Center, Seoul, Korea \\ Department of Neurosurgery, ${ }^{4}$ Samsung Medical Center, Sungkyunkwan University School of Medicine, Seoul, Korea \\ Department of Medical Education, ${ }^{5}$ Sungkyunkwan University School of Medicine, Seoul, Korea \\ Department of Anatomy and Cell Biology, ${ }^{6}$ Sungkyunkwan University School of Medicine, Suwon, Korea
}

Objective : The purpose of this study was to find an optimal delivery route for clinical trials of intrathecal cell therapy for spinal cord injury in preclinical stage.

Methods : We compared in vivo distribution of Cy5.5 fluorescent dye in the spinal cord region at various time points utilizing in vivo optical imaging techniques, which was injected into the lateral ventricle (LV) or cisterna magna (CM) of rats.

Results : Although CM locates nearer to the spinal cord than the LV, significantly higher signal of Cy5.5 was detected in the thoracic and lumbar spinal cord region at all time points tested when Cy5.5 was injected into the LV. In the LV injection Cy5.5 signal in the thoracic and lumbar spinal cord was observed within 12 hours after injection, which was maintained until 72 hours after injection. In contrast, Cy5.5 signal was concentrated at the injection site in the CM injection at all time points.

Conclusion : These data suggested that the LV might be suitable for preclinical injection route of therapeutics targeting the spinal cord to test their treatment efficacy and biosafety for spinal cord diseases in small animal models.

Key Words : Spinal cord injuries · Distribution · Lateral ventricles · Cisterna magna · Fluorescence · Optical imaging.

- Received : September 18, 2017 •Revised : October 9, 2017 •Accepted : October 23, 2017

- Address for reprints : Kyeung Min Joo, M.D., Ph.D.

Department of Anatomy and Cell Biology, Sungkyunkwan University School of Medicine, 2066 Seobu-ro, Suwon 16419, Korea

Tel : +82-31-299-6087, Fax : +82-31-299-6029, E-mail : kmjo0@skku.edu

Sun-Ho Lee, M.D., Ph.D.

Department of Neurosurgery, Samsung Medical Center, Sungkyunkwan University School of Medicine, 81 Irwon-ro, Gangnam-gu, Seoul 06351, Korea Tel : +82-2-3410-2457, Fax : +82-2-3410-0048, E-mail : sobotta72@gmail.com

* Kee-Hang Lee and Hyun Nam contributed equally to this work.

This is an Open Access article distributed under the terms of the Creative Commons Attribution Non-Commercial License (http://creativecommons.org/licenses/by-nc/4.0) which permits unrestricted non-commercial use, distribution, and reproduction in any medium, provided the original work is properly cited. 


\section{INTRODUCTION}

Spinal cord injury (SCI) results in physical discontinuance of ascending and descending neural pathways at damaged regions, which is deteriorated more by secondary damages such as demyelination, inflammation, and gliosis ${ }^{14)}$. Transplantation of exogenous stem cells are considered to be alternative therapeutics since they have regenerative potential that can repopulate damaged neural cells ${ }^{11)}$. Two major types of stem cells such as mesenchymal stem cells and neural stem cells have been widely used to prove preclinical efficacy of stem cells for neurological diseases" ${ }^{9)}$.

To confirm the preclinical efficacy of stem cells, stem cells should be injected into animal models through a verified injection route which can represent the clinical injection route. In many literatures, to evaluate preclinical efficacy of injection, stem cells have been usually injected into the damaged spinal cord regions directly ${ }^{1}$. However, in clinical trials of amyotrophic lateral sclerosis, intrathecal injection of stem cells have been also conducted expecting stem cells to migrate into disease sites via cerebrospinal fluid (CSF) ${ }^{6}$. However, intrathecal injection route would be difficult to be translated in small animals such as mice and rats. To overcome this discordance, it is necessary to find reasonable alternative injection routes which can be translated to clinical injection routes and can verify treatment efficacy of stem cells preclinically.

In this study, to determine ideal clinical injection route for SCI, we established surgical procedures for lateral ventricle (LV) and cisterna magna (CM) injection of stem cells in rats. After injection of a fluorescent dye, Cy5.5 via the LV or CM route, we compared its migration and distribution in the spinal cord at various time points.

\section{MATERIALS AND METHODS}

\section{Animal care}

This study was reviewed and approved by the Institutional Animal Care and Use Committee of Samsung Biomedical Research Institute (20140916001). Animal experiments were conducted in accordance with the protocols that were approved by the Institutional Review Board (IRB) of the Samsung Medical Center (Seoul, Korea) (IRB No. 201403014006).

\section{Surgical procedure for $L V$ injection}

Sprague-Dawley (SD) rats (9-week-old, 250-300 g, female) (Orient Bio., Gapyeong, Korea) were anesthetized by isoflurane (Ifran $^{\mathrm{TM}}$, Hana Pharma, Hwasung, Korea). The rats were fixed in a stereotaxic device (Model 900 small animal stereotaxic instrument, KOPF stereotaxic, Tujunga, CA, USA). The skin on skull was cut about $2 \mathrm{~cm}$ and the external membrane was removed to expose the bregma. We marked injection site (medial/lateral $1.2 \mathrm{~mm}$, anterior/posterior $-0.3 \mathrm{~mm}$, dorsal/ ventral $4 \mathrm{~mm}$ from the bregma) with a Hamilton syringe (22 G, 1702 RN SYR, Small Removable NDL; Hamilton Company, Reno, NV, USA) connected to a stereotaxic device and made a $0.5 \mathrm{~mm}$ diameter hole using a drill (8050-N/18 micro 8V max, Dremel, Racine, WI, USA). 20 nM Cy5.5 (Amersham $\mathrm{CyDye}^{\mathrm{TM}}$ mono-reactive NHS Ester, GE Healthcare, Piscataway, NJ, USA) in $20 \mu \mathrm{L}$ saline was injected using a syringe pump (LEGATO ${ }^{\mathrm{TM}} 111, \mathrm{KD}$ scientific, Holliston, MA, USA). After injection, we waited for five minutes to prevent leaking. The injection needle was raised up carefully by the speed of 1 mm per minute.

\section{Surgical procedure for $\mathrm{CM}$ injection}

SD rats (Orient Bio.) were anesthetized and processed in a stereotaxic device by the same procedure as done in the LV injection. The hair at the skin between the occipital bone and the atlas was removed by an electric shaver. After disinfection with povidone (Green Pharmaceutical Co. Ltd, Jincheon, Korea) and $70 \%$ ethanol, the fronto-cervical area was cut to expose the skull including the upper cervical laminae. The muscles of behind neck were cut to separate from the occipital bone using a surgical blade to expose the CM region. The needle tip of a Hamilton syringe (22G, 1702 RN SYR, Small Removable NDL; Hamilton Company) was inserted diagonally into the $2 / 3$ part of the atlanto-occipital membrane. $20 \mathrm{nM}$ Cy5.5 in $20 \mu \mathrm{L}$ saline was injected at an infusion rate of $2 \mu \mathrm{L} / \mathrm{min}$ using the same procedure as done in the LV injection. After injection, we waited for 5 minutes to prevent leakage of Cy5.5 from the injection hole. After removal of the needle, the injection site was blocked using a bleed blocker (SPONGOSTAN ${ }^{\mathrm{TM}}$ Standard, Ferrosan medical devices, Soborg, Denmark) until leakage was not observed. 


\section{In vivo near-infrared fluorescence (NIRF) imaging of Cy5.5}

Optical NIRF imaging was conducted at 12, 24, 48, and 72 hours after injection of Cy5.5 using a Xenogen IVIS Spectrum system (Caliper Life Science, Hopkinton, MA, USA) according to the previous reports ${ }^{7,17)}$. Identical illumination settings (lamp voltage, filters, f/stop, field of views, and binning) were used for all images. Fluorescence (emission : 720 nm, excitation : $605 \mathrm{~nm}$ ) was measured as photons per second per centimeter squared per steradian $\left(\mathrm{p} / \mathrm{s} / \mathrm{cm}^{2} / \mathrm{sr}\right)$. Quantitative data in each region of interest were acquired and analyzed using Living Image 2.5 software (Caliper Life Science). The head, cervical, thoracic, and lumbar region were defined by from eye to first cervical vertebrae (C1), from $\mathrm{C} 1$ to first thoracic vertebrae (T1), from $\mathrm{T} 1$ to first lumbar vertebrae (L1), and from L1 to first sacral vertebrae (S1), respectively. Representative image of each region was depicted in Fig. 1C. The total flux was measured at each region and the sum of total flux was defined by the sum of total fluxes at all regions.

\section{Statistics}

Data were represented by mean and standard error. Data were analyzed using Student's t-test, two-tailed. $p$-values $<0.05$ were considered statistically significant. Mathematical equations defining the relationships of slopes were derived from bar graph trend lines.

\section{RESULTS}

\section{Establishment of surgical procedures for LV and CM injection}

Optimal surgical procedures for LV and CM injection were developed, which were described in detail in the Materials and Methods section and shown in Fig. 1A and B. For reproducible LV injection, how to find the specific location for a hole on the skull according to the bregma and how to locate threedimensional location of the LV in the brain should be considered. On the contrary, for CM injection, invasiveness and time of surgical procedures need to be minimized and final depth of the needle tip into the CM should be optimized.

\section{In vivo optical imaging of Cy5.5 after LV or CM injection}

To determine the distribution of Cy5.5, we detected the in vivo signal of Cy5.5 at 12, 24, 48, and 72 hours after injection in the head, cervical, thoracic, and lumbar region (Fig. 1C). In the LV injection, we could observe strong distribution of Cy5.5 signal in the thoracic and lumbar region 12 hours after injection (Fig. 2A). The signal intensity of Cy5.5 in the regions maintained until 72 hours after injection. In contrast, most Cy5.5 signal remained the head and cervical region 12 hours after CM injection (Fig. 2B). Even at 72 hours after injection, Cy5.5 signal was concentrated near the injection site, which suggested poor delivery of Cy5.5 to the lower spinal cord (Fig. 2B).

A
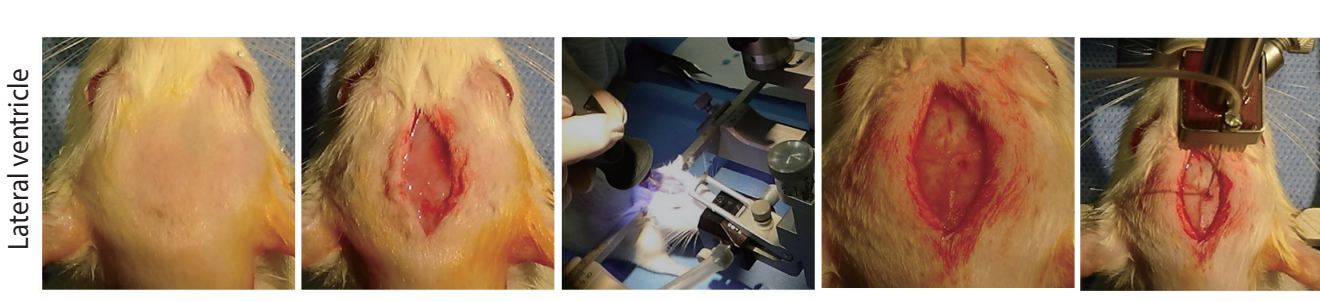

Post-injection
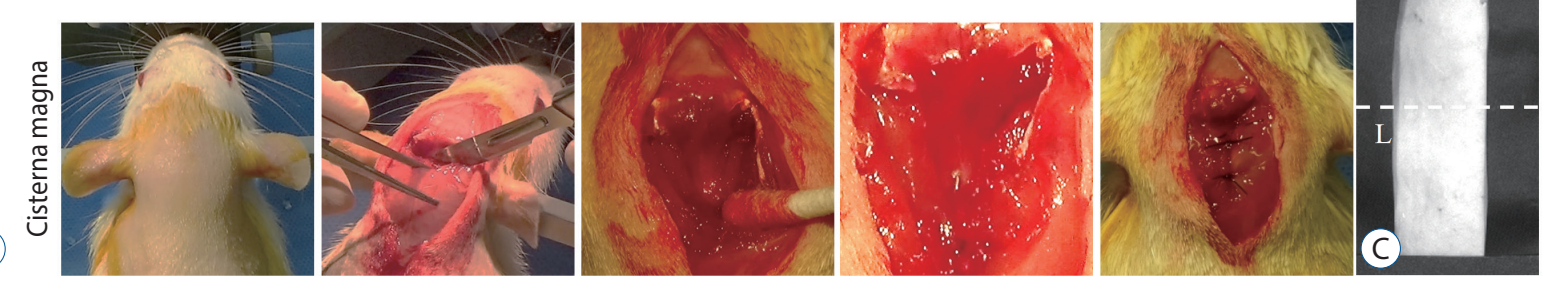

Fig. 1. Surgical procedures for LV and CM injection. Surgical procedures for LV and CM injection were shown in (A) and (B), respectively. A : For LV injection, after anesthesia, rats were fixed with a stereotaxic device and cut to expose the bregma. After making a hole with a drill, a needle was injected into the LV. B : For CM injection, after anesthesia, rats were fixed with a stereotaxic device. After surgery to expose CM, Cy5.5 was injected into CM. C : Each region of interest to measure Cy 5.5 was demonstrated. $\mathrm{H}$ : head, $\mathrm{C}$ : cervical, $\mathrm{T}$ : thoracic, L : lumbar, LV : lateral ventricle, CM : cisterna magna. 


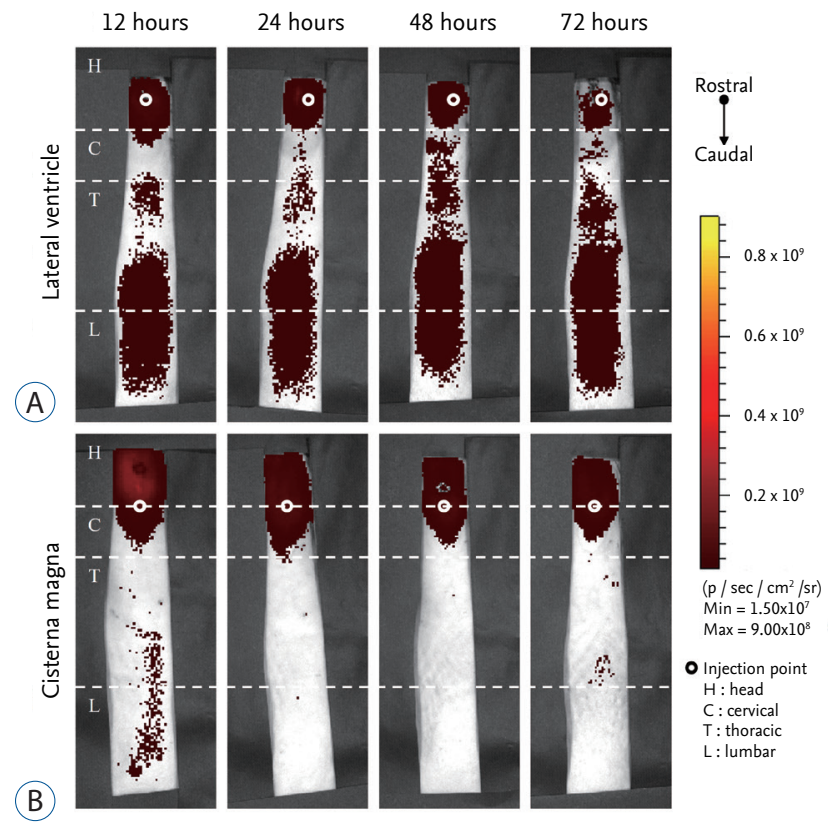

(A)

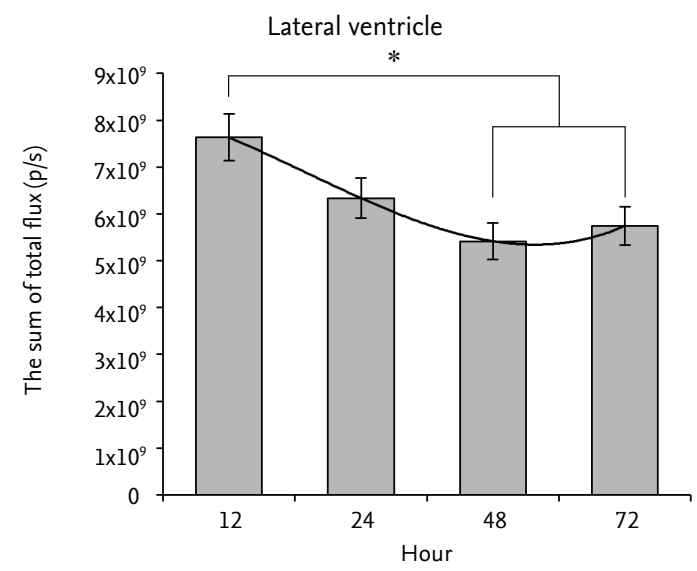

(C)

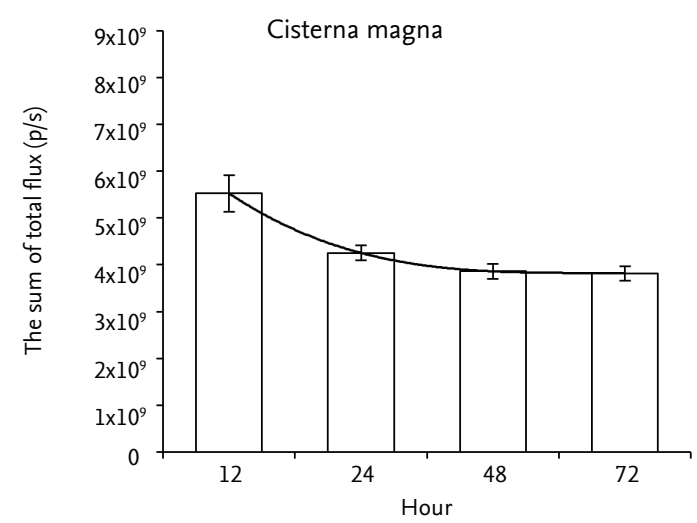

(B)
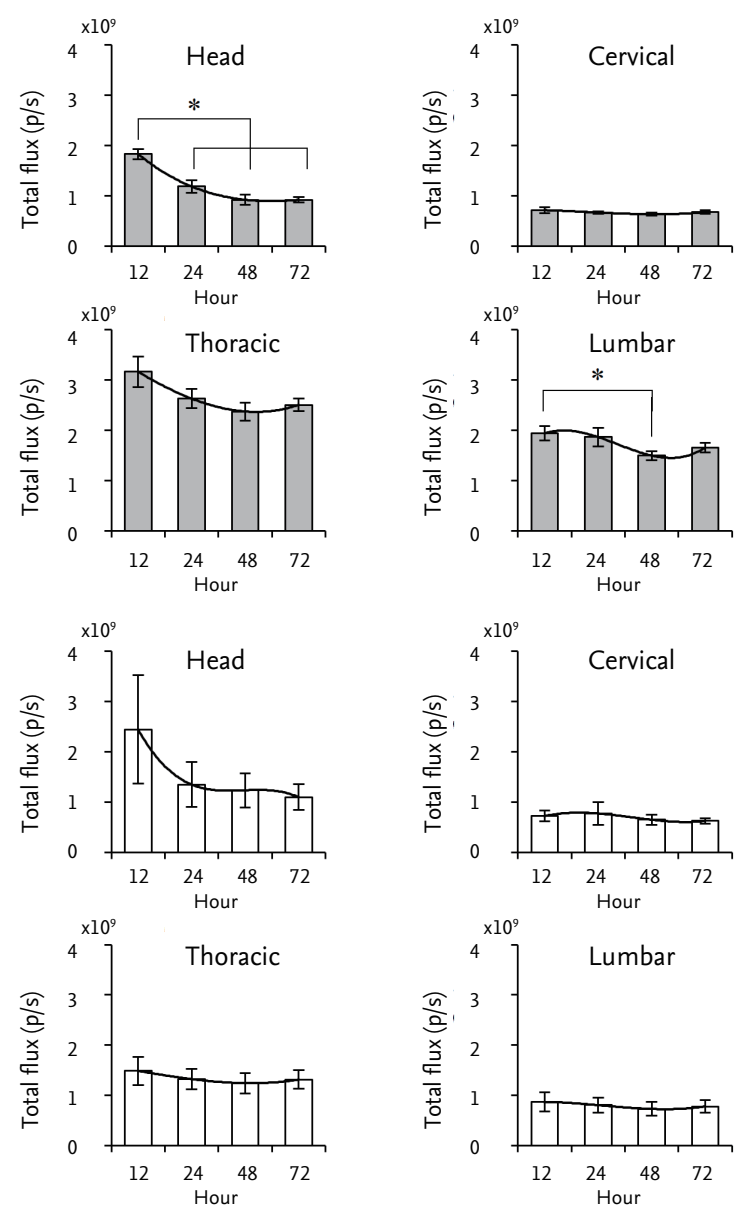

Fig. 3. Quantitation of the in vivo signal intensity of Cy5.5 after LV and CM injection. The total flux of Cy5.5 signal was quantitated at each region of interest. A : After LV injection, the sum of total fluxes was shown at each time point. B : The total flux of Cy5.5 at each region of interest was shown at various time points. C : After CM injection, the sum of total fluxes was shown at each time point. D : The total flux of Cy5.5 at each region of interest was shown at various time points. ${ }^{*} p<0.05$. LV : lateral ventricle, $\mathrm{CM}$ : cisterna magna. 


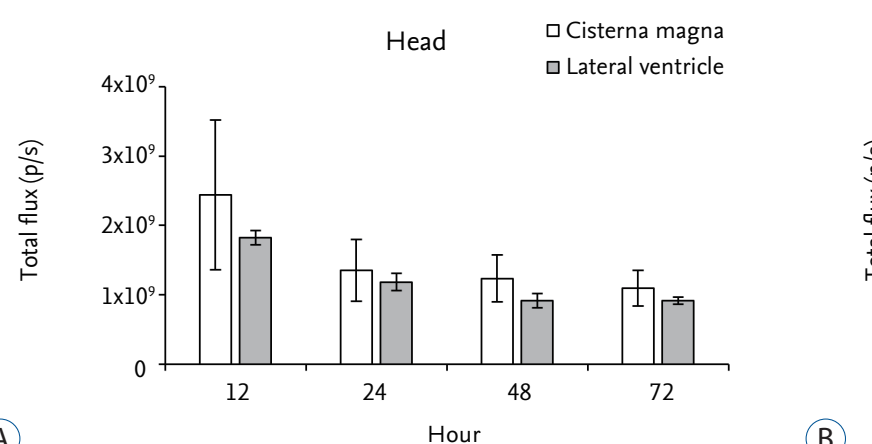

(A)

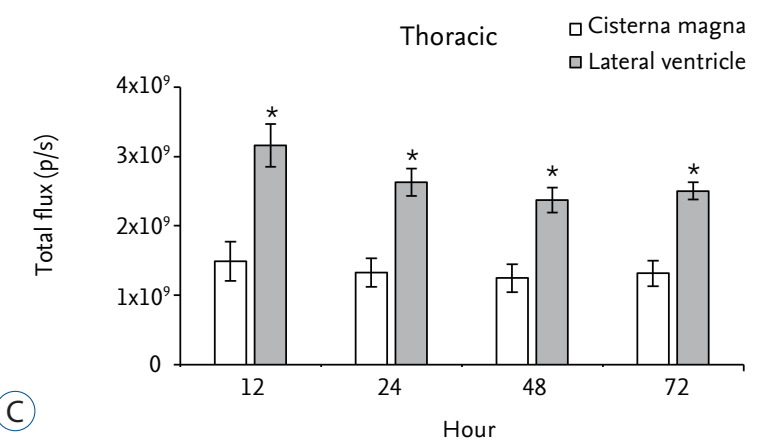

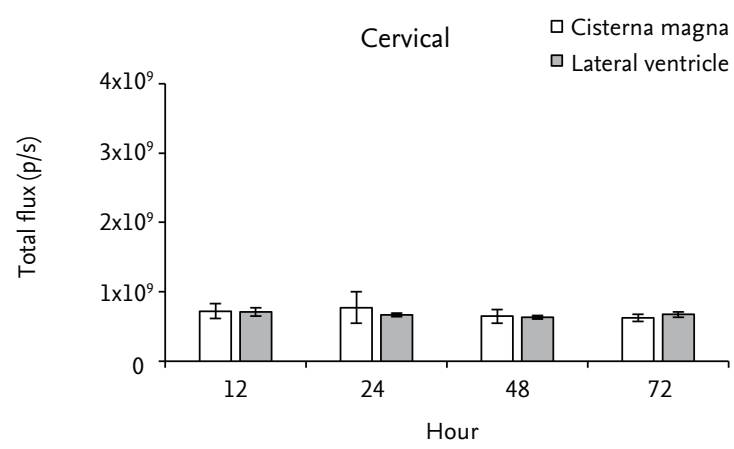

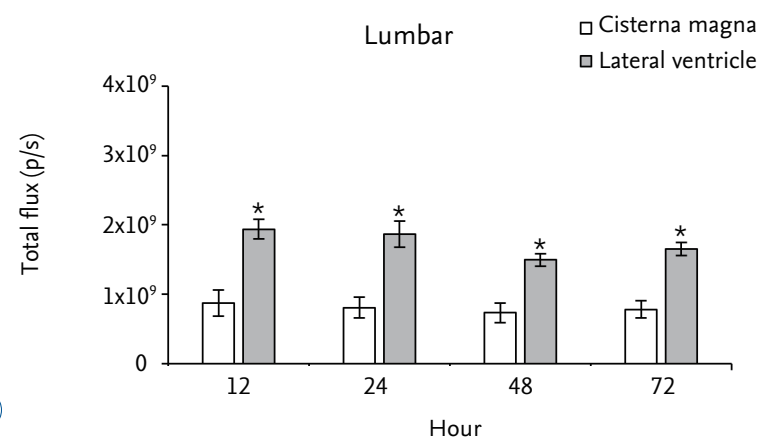

Fig. 4. Comparison of the in vivo signal intensity of Cy5.5 according to the injection route at each region of interest. The regional total flux of Cy5.5 at each time point was compared between LV and CM injection route. A : In head region, CM injection showed slightly higher signal intensity than that of the LV injection but not statistically significant. B : In cervical region, both injection methods showed similar signal intensity. $C$ and $D:$ In thoracic and lumbar region, LV injection showed significantly higher signal intensity than CM injection. ${ }^{*} p<0.05$. LV : lateral ventricle, CM : cisterna magna.

\section{Quantitation of in vivo Cy5.5 signal}

The signal intensity of Cy5.5 was quantitated and compared. In the LV injection, total flux of Cy5.5 signal decreased in a time-dependent manner and there were significant decreases at 48 hours and 72 hours after injection (Fig. 3A). When regional Cy5.5 signal was analyzed, there was no significant change in Cy5.5 signal in the cervical region (Fig. 3B). The Cy5.5 signal in the head, thoracic, and lumbar region was highest at 12 hours after injection, which decreased until 48 hours after injection (Fig. 3B). In the CM injection, the total flux of Cy5.5 signal also decreased in a time-dependent manner (Fig. 3C). Although Cy5.5 signal decreased at the head region, significant change in Cy5.5 signal was not observed in the cervical, thoracic, and lumber region all the time point after injection (Fig. 3D).

\section{Distribution difference of Cy5.5 according to injection routes}

We compared Cy5.5 signal in each region according to injection routes. In the head region, Cy5.5 signal of the CM in- jection was slightly higher than that of the LV injection although it was not statistically significant (Fig. 4A). In the cervical region, Cy5.5 signal was similar between the LV and $\mathrm{CM}$ injection at all-time points (Fig. 4B). In the thoracic and lumbar region, Cy5.5 signals of the LV injection were significantly higher than those of the $\mathrm{CM}$ injection at all-time points (Fig. 4C and D). These data suggested that LV injection might be better than CM injection for the distribution of injected materials to the lower spinal cord.

\section{DISCUSSION}

CSF is a body fluid produced in the choroid plexuses of the ventricles of the brain. The functions of CSF include mechanical cushion and chemical buffer for the central nervous system (CNS). In addition, CSF has vital functions in the autoregulation of cerebral blood flow ${ }^{2}$. The flow of CSF provides a delivery route of materials to various regions of CNS including the spinal cord. To determine the flow of CSF, various types 
of substances have been used in vivo ${ }^{3,13)}$. In this study, we optimized surgical procedures for CM and LV injection of Cy5.5 into CSF of rats and evaluated spatiotemporal distribution of Cy5.5 within CSF of rats.

The CM includes three principal openings between the ventricular system in the brain and in the subarachnoid space outside of the brain. CSF produced in the ventricle flows into the CM via the lateral and median apertures and then diffuse into the subarachnoid space of the brain and spinal cord. Since the CM is located underneath the cerebellum and behind of the dorsal surface of the medulla oblongata, it can be accessed from the outside relatively easily in humans. Therefore, the CM has been considered as an optimal route to collect $\mathrm{CSF}^{5,12)}$ or inject materials into CSF. Previously, various types of animals including rat, rabbit, dog, and monkey have been tested for CM injection ${ }^{8,10,15,16)}$.

In medium and large size animals CM injection has some advantages compared to LV injection, which requires less invasive procedures and simpler surgical instruments ${ }^{4)}$. However, it is difficult to optimize CM injection in small animal species such as mouse and rat. Moreover, detailed surgical procedures for CM injection in small animals were not described fully in many previous studies using CM injection ${ }^{133}$. To increase accuracy and reproducibility of CM injection in small animals, injecting instruments which can deliver various materials into the CM have been developed ${ }^{4}$. To assure accuracy and reproducibility, rats were fixed in a stereotaxic device and CM was exposed and visualized by surgery in this study. However, the distribution of Cy5.5 was limited to the head and cervical region until 72 hours after injection. Although we utilized surgical techniques to prevent leakage of Cy5.5, it cannot be excluded that Cy5.5 was leaked due to the positive pressure of the subarachnoid space and surgical dead spaces.

The LVs are the two big cavities of the ventricular system in the brain, which are filled with CSF. The ventricular system consists of the lateral ventricles, third ventricle, and fourth ventricle. There are several foramina acting as channels that connect the ventricles. This connection makes CSF flow from the lateral ventricles to the central canal and subarachnoid space of the spinal cord. Based on the circulation of CSF, LV injection has been widely applied for the delivery of materials into the CNS in various animal species ${ }^{2,3)}$.

In small animals, LV injection has advantages compared to
CM injection; LV injection needs simple surgical techniques, has little leakage of CSF, and makes lesser bleeding. Moreover, the simple surgical procedure enables repeated injection via LV route. Our results were also supportive for the previous reports. In addition, we observed that Cy5.5 fluorescent dye located in the thoracic and lumbar spinal cord region within 12 hours when it was injected into the LV. With a stereotaxic device, Cy5.5 could be injected into the LV accurately which resulted in its fast circulation in the CNS. Based on the advantages of the LV injection, LV could be utilized as a reliable route for the delivery of materials to the spinal cord in the small animals including rats. However, our results using Cy5.5 may not be translated into stem cells directly because stem cells have different molecular size, solubility in water, and dynamics within CSF compared to Cy5.5.

\section{CONCLUSION}

In this study, we injected Cy5.5 fluorescent dye into the LV or $\mathrm{CM}$ of rats and compared the in vivo distribution of Cy5.5 in the spinal cord regions. Since significantly more Cy5.5 in vivo signal was detected in the LV injection than the CM injection, we concluded that the LV injection might be more suitable than the CM injection to determine preclinical treatment efficacy or biosafety of stem cells for spinal cord diseases in small animal models.

\section{CONFLICTS OF INTEREST}

No potential conflict of interest relevant to this article was reported.

\section{INFORMED CONSENT}

Informed consent was obtained from all individual participants included in this study.

\section{- Acknowledgements}

This research was supported by grants from Ministry of Food and Drug Safety in 2018 (18172MFDS182) and National Research Foundation (NRF-2016R1D1A1B03933833 and 
NRF-2016R1A5A2945889).

\section{References}

1. Björklund $A$, Lindvall 0 : Cell replacement therapies for central nervous system disorders. Nat Neurosci 3 : 537-544, 2000

2. Boulton $M$, Flessner $M$, Armstrong $D$, Mohamed R, Hay J, Johnston $M$ : Contribution of extracranial lymphatics and arachnoid villi to the clearance of a CSF tracer in the rat. Am J Physiol 276 : R818-R823, 1999

3. Bradbury MW, Lathem W : A flow of cerebrospinal fluid along the central canal of the spinal cord of the rabbit and communications between this canal and the sacral subarachnoid space. J Physiol 181 : 785-800, 1965

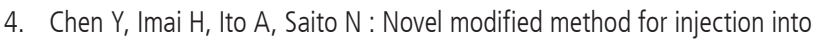
the cerebrospinal fluid via the cerebellomedullary cistern in mice. Acta Neurobiol Exp (Wars) 73 : 304-311, 2013

5. Consiglio $A R$, Lucion $A B$ : Technique for collecting cerebrospinal fluid in the cisterna magna of non-anesthetized rats. Brain Res Brain Res Protoc 5 : 109-114, 2000

6. Feldman EL, Boulis NM, Hur J, Johe K, Rutkove SB, Federici T, et al. : Intraspinal neural stem cell transplantation in amyotrophic lateral sclerosis: phase 1 trial outcomes. Ann Neurol 75 : 363-373, 2014

7. Gao GH, Park MJ, Li Y, Im GH, Kim JH, Kim HN, et al. : The use of pHsensitive positively charged polymeric micelles for protein delivery. Biomaterials 33 : 9157-9164, 2012

8. Johnston M, Zakharov A, Koh L, Armstrong D : Subarachnoid injection of Microfil reveals connections between cerebrospinal fluid and nasal lymphatics in the non-human primate. Neuropathol Appl Neurobiol $31: 632-640,2005$

9. Kim SU, de Vellis J : Stem cell-based cell therapy in neurological dis- eases: a review. J Neurosci Res 87 : 2183-2200, 2009

10. Koedel U, Bernatowicz A, Frei K, Fontana A, Pfister HW : Systemically (but not intrathecally) administered IL-10 attenuates pathophysiologic alterations in experimental pneumococcal meningitis. J Immunol 157 : 5185-5191, 1996

11. Lindvall O, Kokaia Z, Martinez-Serrano A : Stem cell therapy for human neurodegenerative disorders-how to make it work. Nat Med 10 Suppl : S42-S50, 2004

12. Liu $L$, Duff $K$ : A technique for serial collection of cerebrospinal fluid from the cisterna magna in mouse. J Vis $\operatorname{Exp}(21): 960,2008$

13. Proescholdt MG, Hutto B, Brady LS, Herkenham M : Studies of cerebrospinal fluid flow and penetration into brain following lateral ventricle and cisterna magna injections of the tracer [14C]inulin in rat. Neuroscience 95 : 577-592, 2000

14. Rowland JW, Hawryluk GW, Kwon B, Fehlings MG : Current status of acute spinal cord injury pathophysiology and emerging therapies: promise on the horizon. Neurosurg Focus 25 : E2, 2008

15. Shirao S, Fujisawa H, Kudo A, Kurokawa T, Yoneda H, Kunitsugu I, et al. : Inhibitory effects of eicosapentaenoic acid on chronic cerebral vasospasm after subarachnoid hemorrhage: possible involvement of a sphingosylphosphorylcholine-rho-kinase pathway. Cerebrovasc Dis $26: 30-37,2008$

16. van der Flier M, Coenjaerts FE, Mwinzi PN, Rijkers E, Ruyken M, Scharringa J, et al. : Antibody neutralization of vascular endothelial growth factor (VEGF) fails to attenuate vascular permeability and brain edema in experimental pneumococcal meningitis. J Neuroimmunol 160 : 170-177, 2005

17. Yang HY, Jang MS, Li Y, Lee JH, Lee DS : Multifunctional and redoxresponsive self-assembled magnetic nanovectors for protein delivery and dual-modal imaging. ACS Appl Mater Interfaces 9 : 19184-19192, 2017 